\title{
Método de Reducción de Incertidumbre Basado en Algoritmos Evolutivos y Paralelismo Orientado a la Predicción y Prevención de Desastres Naturales
}

\section{Uncerainty Reduction Method Based on Evolutionary Algorithms and Parallelism Applyed to Natural Disasters Prediction and Prevention}

Presentación: 28/08/2020

\section{Doctorando:}

Miguel Méndez Garabetti

Laboratorio de Investigación en Cómputo Paralelo Distribuido, Universidad Tecnológica Nacional, Facultad Regional Mendoza Argentina

mmendez@mendoza-conicet.gob.ar

\section{Director/a:}

\section{Germán Bianchini}

\section{Co-director/a:}

\section{Verónica Gil Costa / Paola Caymes Scutari}

\section{Resumen}

La falta de precisión en los parámetros de entrada en cualquier modelo científico suele ser habitual debido a la imposibilidad de cuantificar todos los factores que afectan al modelo en tiempo real. Esto puede producir consecuencias dramáticas en la salida del mismo si se trata de un sistema crítico, tal como lo es en el caso particular de la predicción de fenómenos naturales. No obstante, también deben sumarse las limitaciones impuestas por los propios modelos, las restricciones que agregan las soluciones numéricas y las provenientes de las propias implementaciones y versiones informáticas. Por tal motivo, esta investigación se ha centrado en el desarrollo de métodos informáticos que se enfoquen en el tratamiento de la incertidumbre de dichos valores de entrada para lograr así una predicción lo más confiable posible por parte del modelo. En base a esto, se han desarrollado dos métodos de reducción de incertidumbre que han sido validados en la predicción del comportamiento de incendios forestales. Dichos métodos utilizan como componentes: Análisis Estadístico, Algoritmos Evolutivos Paralelos, Computación de Alto Desempeño e Información Satelital. El análisis estadístico ofrece la posibilidad de estudiar tendencias en el comportamiento del sistema bajo diversas condiciones, los algoritmos evolutivos paralelos permiten orientar la búsqueda brindando una predicción más precisa, la computación paralela/distribuida brinda infraestructura necesaria para poder llevar a cabo el procesamiento en cantidad de tiempo razonable y la información satelital permite aplicar estos métodos situaciones de emergencia real. Cada uno de estos componentes son indispensables para lograr valores aceptables tanto en términos de calidad de predicción como términos de tiempo de respuesta de los métodos propuestos,

Palabras clave: reducción de incertidumbre, predicción, paralelismo, algoritmos evolutivos, desastres naturales 


\begin{abstract}
The imprecision in the input parameters in any scientific model is generally due to the impossibility of quantifying all factors that affect the model in real time. This can produce dramatic consequences in the output of the model, and even more if it is a critical system such is as the natural disasters prediction. However, the limitations of the models themselves, the numerical solutions restrictions and those coming from the implementations and computer versions must also be considered. For this reason, this research has focused on the development of uncertainty reduction methods to achieve the most reliable prediction possible. Due to this, two uncertainty reduction methods validated in the forest fire behavior prediction have been developed. These methods use as components: Statistical Analysis, Parallel Evolutionary Algorithms, High Performance Computing and Satellite Information. Statistical analysis allows studying trends in the behavior of the system under various condition, parallel evolutionary algorithms allow searching for a more accurate prediction, parallel computing provides the necessary infrastructure to be able to carry out the processing in a reasonable amount of time, and satellite information allows these methods to be applied in real emergencies. Each of these components are essential to achieve better prediction quality and response time of the proposed methods.
\end{abstract}

Keywords: uncertainty reduction, prediction, parallelism, evolutionary algorithms, natural disasters

\title{
Introducción
}

Los modelos se han convertido en uno de los principales instrumentos de la ciencia moderna, éstos suelen utilizarse en la representación de gran cantidad de sistemas físicos, inclusive en el ámbito de la ciencia ambiental para efectuar predicciones. La modelización puede ser vista como el establecimiento de relaciones semánticas entre la teoría y los fenómenos u objetos. Generalmente, los modelos representan alguna situación real de manera incompleta e inexacta; esto permite realizar estudios de sistemas complejos de forma aproximada. Usualmente, los modelos deben ser alimentados con parámetros de entrada, los cuales brindan la información necesaria con la cual es posible determinar las condiciones iniciales del fenómeno bajo estudio, y de esta manera estimar sus posibles variaciones en el tiempo.

La falta de precisión en los parámetros de entrada en cualquier modelo científico suele ser habitual debido a la imposibilidad de cuantificar todos los factores que afectan al modelo en tiempo real. Esto puede producir consecuencias dramáticas en la salida del mismo si se trata de un sistema crítico, como lo es la predicción. Esta problemática, que surge a partir de la falta de precisión o exactitud en los parámetros de entrada de un modelo, se conoce como incertidumbre. Como bien se menciona en (Ishigami y Homma 1990): “cuando se analizan sistemas complejos, los resultados calculados incluyen incertidumbres debidas a los propios modelos informáticos, como así también a las existentes en los valores de las variables de entrada o los parámetros utilizados en el análisis. Debido a esto, es importante no solo cuantificar las incertidumbres de salida sino también identificar las variables de entrada dominantes que contribuyen a las incertidumbres de salida". En base a esto, se puede decir que en cualquier ámbito donde se realice algún tipo de predicción existirá mayor o menor grado de incertidumbre (tanto se trate de un modelo económico, meteorológico o natural) y con ello el esfuerzo por intentar minimizar sus efectos negativos. Dicho en otras palabras, ante la presencia de incertidumbre (principal fuente de imprecisión en todo sistema de predicción), es necesario intentar eliminarla, o en el mejor de los casos lograr reducirla. En la literatura se utiliza el término Reducción de Incertidumbre o Métodos de Reducción de Incertidumbre para hacer referencia a aquellas técnicas o herramientas que de alguna manera permiten reducir los efectos causados por la falta de precisión en cualquier variable o factor de un sistema de predicción (Ishigami y Homma 1990).

En el contexto de esta investigación se define un Método de Reducción de Incertidumbre (MRI) como un sistema computacional que tiene como propósito mejorar la calidad de salida de un modelo determinado, de forma independiente a los datos con que dicho modelo haya sido alimentado. Esto se logra mediante algún tipo de pre-procesamiento y/o pos-procesamiento de los datos de entrada y/o salida del modelo. Las funciones llevadas a cabo por un MRI no deben interferir con las operaciones internas del modelo bajo análisis, es decir, este último debe ser tratado como una caja negra. 
En este sentido, la presente investigación contribuye al desarrollo de métodos de reducción de incertidumbre capaces de asistir y participar en el proceso de resolución de problemas de alta magnitud y complejidad computacional, tal como la predicción y prevención de desastres naturales. Este tipo de problemas puede ser clasificado en la categoría de Grand Challenge Problems (Wilkinson y Allen 1999), es decir, problemas de alta complejidad, y a la vez importantes (desde el punto de vista social y económico) donde se requieren varios órdenes de magnitud de recursos para resolverlos. Los métodos desarrollados, si bien han sido concebidos como generales (ya que podrían ser aplicados a diferentes fenómenos naturales con características de propagación), han sido validados tras su aplicados a la predicción del comportamiento de incendios forestales.

\section{Desarrollo}

En el contexto de este trabajo, se desarrollaron dos métodos que centran sus esfuerzos en minimizar el problema de la incertidumbre en los parámetros de entrada utilizando técnicas de: simulación (Arahal, Berenguel Soria, y Rodríguez Díaz 2006), análisis estadístico (Montgomery y Runger 2014), computación de alto rendimiento (Buyya 1999) y optimización metaheurística (Blum, Roli, y Sampels 2008; Talbi 2009). En el desarrollo de tales métodos también se involucran diferentes aspectos relacionados con: modelos y paradigmas de computación paralela, hibridación de diferentes tecnologías de paralelización (MPI, OpenMP y CUDA), simuladores de incendios forestales, entre otros.

De forma sintética se puede describir el funcionamiento de estas metodologías de la siguiente manera: la simulación se utiliza en el centro de nuestro sistema en donde se llevan a cabo múltiples ejecuciones de un simulador de comportamiento de incendios forestales, cada una con diferentes configuraciones de parámetros de entrada. Tales configuraciones de parámetros representan un escenario diferente, al cual se le asocia un valor de aptitud en cada paso de predicción, un valor de aptitud en cada paso de predicción, los cuales se optimizan mediante diferentes técnicas metaheurísticas, que se ejecutan de forma concurrente. El paralelismo se vuelve necesario debido al costo computacional de estos procesos y, también, debido a que los resultados deben ser obtenidos en el menor tiempo posible. Finalmente, la estadística permite estimar un patrón de comportamiento del incendio, basado en un análisis de probabilidad considerando la totalidad de las simulaciones llevadas a cabo, esperando que dicho patrón permita realizar una predicción inmediata futura. Estas técnicas, integradas en los métodos propuestos, permiten incrementar la calidad de predicción frente a las limitaciones ya conocidas.

Los métodos desarrollados han sido denominados como: "Sistema Estadístico Evolutivo con Modelo de Islas" (ESS-IM, por sus siglas en inglés) (Mendez-Garabetti et al. 2017; M. Méndez-Garabetti et al. 2016) y "Sistema Estadístico Híbrido Evolutivo con Modelo de Islas" (HESS-IM, por sus siglas en inglés) (Miguel Méndez-Garabetti et al. 2017). Ambos se corresponden con métodos de reducción de incertidumbre desarrollados para su aplicación en la predicción de desastres naturales. En este trabajo ambos métodos han sido aplicados a la predicción del comportamiento de incendios forestales. Para este propósito se ha utilizado un modelo de simulación, en este caso se corresponde con el modelo de comportamiento de incendios forestales de Rothermel (Rothermel 1972). Puntualmente se trata del simulador de incendios forestales implementado en ANSI C mediante la librería fireLib (Collin D. Bevins 1996).

Es importante remarcar que esta investigación también abordó el diseño de un Sistema Integrado de Detección, Alerta Temprana y Predicción de Incendios Forestales incluyendo a uno de los métodos de reducción de incertidumbre desarrollados (HESS-IM), utilizado como sistema de predicción de comportamiento de incendios forestales. Y la integración con dos subsistemas: a) módulo de generación automática de mapas para su posterior tratamiento, y b) red (WSN/IoT, Wireless Sensor Network/Internet of Things) para la detección temprana de incendios forestales. El enfoque propuesto coincide con los Sistemas Dinámicos de Aplicaciones Guiadas por Datos (DDDAS, Dynamic Data-Driven Application Systems) (Darema 2004), los cuales tratan de aprovechar al máximo la unión de los métodos de adquisición de datos, la computación y simulación en el desarrollo de sistemas. La creación de un DDDAS requiere la comprensión sobre el uso de sensores remotos, diferentes niveles de computación (por ejemplo, procesadores embebidos, supercomputadoras, redes, seguridad de datos, modelado matemático/computacional, problemas inversos, teoría de control, visualización, entre otros). En este sentido se desarrolló un subsistema para la generación 
automática de mapas que incendios a partir de imágenes satelitales MODIS (Moderate-Resolution Imaging Spectroradiometer) (NASA 2018), permitiendo operar con incendios forestales naturales.

Desde el punto de vista de los resultados obtenidos se puede resumir que la hibridación implementada en HESS-IM utilizando tres metaheurísticas poblacionales evolutivas ha permitido mejorar el optimizador del método incrementando la calidad de predicción del método. La hibridación se ve mejorada utilizando un esquema de migración basado en topología de anillo entre diferentes metaheurísticas utilizando una configuración de parámetros clásica. La metodología es validada aplicando la misma sobre casos de quemas controladas como así también en casos de incendios forestales. La implementación CPU-GPU ha permitido mejorar también el rendimiento del método en términos de tiempo de ejecución, siendo este un factor de suma importancia en los sistemas abocados a resolver problemáticas con gran impacto social.

\section{Conclusiones}

En esta investigación se ha trabajado en el tratamiento de la reducción de incertidumbre en relación a los métodos de predicción y prevención de desastres naturales. Puntualmente se ha abordado como caso de estudio la problemática de los incendios forestales, uno de los más grandes problemas que afecta extensas regiones a lo largo del mundo. El problema en cuestión ha sido tratado desde diferentes perspectivas. Por un lado, el enfoque principal de este trabajo consistió en el desarrollo de métodos de reducción de incertidumbre que puedan ser utilizados para predecir el comportamiento de este fenómeno. De forma conjunta, se ha avanzado en otras aristas, como el diseño de un sistema integral de detección, alerta temprana y predicción de incendios forestales, el cual podría brindar asistencia en la lucha y mitigación de incendios, como así también en la prevención de los mismos. Este sistema integra los métodos propuestos, con el uso de redes inalámbricas de sensores y un sistema de generación automática de mapas alimentado por imágenes satelitales. Esta línea de trabajo podría hacer posible que este tipo de metodologías llegasen a ser implementadas y adoptadas por entes gubernamentales, permitiendo de esta manera minimizar los daños causados por este fenómeno.

En este trabajo se han propuesto y desarrollado dos métodos de reducción de incertidumbre capaces de asistir y participar en el proceso de resolución de problemas de alta magnitud y complejidad computacional, tal como la predicción y prevención de desastres naturales. En este sentido, los métodos desarrollados son: a) Sistema Estadístico Evolutivo con Modelo de Islas (ESSIM), y b) Sistema Estadístico Híbrido Evolutivo con Modelo de Islas (HESS-IM), los cuales han logrado mejoras considerables en relación a la calidad de las predicciones generadas y así también en términos de tiempo de respuesta, inclusive tras su aplicación con incendios forestales de gran escala.

Estos métodos han sido diseñados para poder aplicarse a otros fenómenos naturales con características de propagación (como avalanchas, deslizamientos de tierra, deslaves, inundaciones, entre otros). Debido a que estos fenómenos suelen causar gran impacto en las comunidades, la flora y la fauna, el ecosistema, entre otros, los sistemas de predicción deben proporcionar respuesta

en el menor tiempo posible (sistemas altamente críticos). Por estos motivos, los métodos propuestos han sido desarrollados utilizando capacidades de alto rendimiento.

Finalmente, se describen algunas líneas de trabajo futuro que se desprenden del presente trabajo:

1. Llevar a cabo estudios de sintonización que permitan sacar el máximo provecho a los métodos diseñados. En este sentido, es de interés llevar a cabo estudios complementarios de sintonización, analizando cómo afectan (tanto en calidad de predicción como en tiempo de procesamiento) la variación de la totalidad de los parámetros de forma simultánea (parámetros de cada metaheurística). También, evaluar el uso de técnicas de metaoptimización, sintonización dinámica, donde se evalúen los efectos de las variaciones de cada parámetro y las interdependencias entre ellos de forma automática (Camilleri y Montebello 2017). 
2. Explorar otras alternativas de hibridación entre metaheurísticas. En la literatura existen numerosos antecedentes donde se utilizan metaheurísticas híbridas basadas en métodos poblacionales y de trayectoria que han sido aplicados de forma exitosa en gran variedad de problemas.

3. Optimizar el funcionamiento de los métodos para la operación de casos de incendios reales. Se considera importante enfocar los esfuerzos de trabajo en la optimización de los métodos para su funcionamiento en casos de incendios reales. Ya sea añadiendo nuevas funcionalidades, modelos, datos, o mediante estudios de sintonización. De esta manera se espera poder aproximar, en mejor medida, las predicciones a la realidad.

4. Desarrollar plataforma de visualización: Pensando en herramientas para la toma de decisiones, es fundamental contar con un módulo que permita visualizar y/o monitorear el frente de fuego a medida que las predicciones se realizan.

5. Culminar la integración entre los métodos de predicción y el sistema de alerta temprana y el módulo de generación automática de mapas. Esta línea se encuentra con un alto grado de avance y se considera oportuno culminar este desarrollo. La funcionalidad final permitirá que a partir de una alerta (ya sea proviniendo de la WSN o a partir de la detección de un foco de calor MODIS) se inicie el proceso de seguimiento del evento. Generando mapas adaptados a la entrada del método de predicción. De esta manera el método llevará a cabo la predicción de forma automática entregando la información de predicción a los tomadores de decisión.

\section{Referencias}

Arahal, Manuel R., Manuel Berenguel Soria, y Francisco Rodríguez Díaz. 2006. Técnicas de predicción con aplicaciones en ingeniería. Secretariado de Publicaciones, Universidad de Sevilla.

Blum, Christian, Andrea Roli, y Michael Sampels. 2008. Hybrid metaheuristics--an emerging approach to optimization. $1^{\mathrm{a}}$ ed. ed. Michael Blum, Christian, Roli, Andrea, Sampels. Berlin Heidelberg: Springer-Verlag Berlin Heidelberg.

Buyya, R. 1999. High Performance Cluster Computing: Architectures and Systems. Prentice Hall, PTR, NJ, USA.

Camilleri, Michel, y Matthew Montebello. 2017. "Optimising the meta-optimiser in machine learning problems". ACM International Conference Proceeding Series Part F1283: 15-22. http://dl.acm.org/citation.cfm?doid=3055635.3056613 (17 de marzo de 2020).

Collin D. Bevins. 1996. fireLib User Manual and Technical Reference.

Darema, Frederica. 2004. 3038 LNCS Dynamic Data Driven Applications Systems: A New Paradigm for Application Simulations and Measurements.

Ishigami, T, y T Homma. 1990. "An importance quantification technique in uncertainty analysis for computer models". En Proceedings of the ISUMA 90', First International Symposium on Uncertainty Modelling and Analysis, IEEE Comput. Soc. Press, 398-403.

Mendez-Garabetti, M., G. Bianchini, P. Caymes-Scutari, y M.L. Tardivo. 2017. "ESS-IM applied to forest fire spread prediction: Parameters tuning for a heterogeneous configuration". En Proceedings - International Conference of the Chilean Computer Science Society, SCCC,.

Méndez-Garabetti, M., G. Bianchini, P. Caymes-Scutari, y M.L. Tardivo. 2016. "Increase in the quality of the prediction of a computational wildfire behavior method through the improvement of the internal metaheuristic". Fire Safety Journal 82: 49-62.

Méndez-Garabetti, Miguel et al. 2017. "Hybrid-Parallel Uncertainty Reduction Method Applied to Forest Fire Spread Prediction". Journal of Computer Science and Technology, ISSN-e 1666-6038, Vol. 17, No. 1, 2017, págs. 12-19 17(1): 12-19. 
Montgomery, D C, y G C Runger. 2014. 6th Editio Applied Statistics and Probability for Engineers. Limusa Wiley \& Sons, New Jersey.

NASA. 2018. "MODIS Web”. https://modis.gsfc.nasa.gov/ (23 de noviembre de 2018).

Rothermel, R C. 1972. II A mathematical model for predicting fire spread in wildland fuels. Res. Pap. INT-115, US Dept. of Agric., Forest Service, Intermountain Forest and Range Experiment Station. (Ogden, UT.).

Talbi, El-Ghazali. 2009. Metaheuristics : from design to implementation. John Wiley \& Sons.

Wilkinson, Barry., y C. Michael. Allen. 1999. Parallel Programming: Techniques and Applications Using Networked Workstations and Parallel Computers. Pearson/Prentice Hall. 